\title{
IdeTo: Spreadsheets for Calculation and Analysis of Area Under the Disease Progress Over Time Data
}

\author{
Ivan Simko ${ }^{\dagger}$
}

U.S. Department of Agriculture, Agricultural Research Service, Crop Improvement and Protection Research Unit, Salinas, CA

\begin{abstract}
When disease ratings are obtained over time, area under the disease progress metrics such as area under the disease progress curve (AUDPC) and area under the disease progress stairs (AUDPS), which allow integration of these measures into a single value, have found use for both survey or replicated experiment data. IdeTo, an Excel-based calculator, computes AUDPC, AUDPS, and their standardized and relative values for up to 200 individuals evaluated at 200 timepoints. In addition to the areas, descriptive statistics are provided for the group of individuals (e.g., accessions and replicates), and both Pearson and Spearman correlation coefficients between the areas and other traits of interest. Graphs are provided to visualize the progression of disease scores over time, distribution of AUDPC and AUDPS values in the dataset, and their linear correlation with the traits of interest.
\end{abstract}

Plant scientists working with measures of plant disease intensity collected over time, commonly known as disease progress, have developed methods for mathematically summarizing and analyzing data, where the fit of population dynamic models has been centered (Madden et al. 2007). In addition to being a useful alternative to modeling when patterns of disease progress do not fit simple growth models (Xu 2006), area under the disease progress curve (AUDPC) combines repeated (in time) observations of disease into a single value (Van der Plank 1963). The original method uses a simple trapezoidal rule to calculate the area defined by monotonically increasing time series data plotted on the $x$-axis and the disease ratings plotted on the $y$-axis. AUDPC has been considered of value for assessing quantitative disease resistance in plants and for evaluating disease management practices (Jeger 2004; Jeger and Viljanen-Rollinson 2001; Lebeda and Jendrulek 1988). It has been pointed out, however, that the trapezoidal method systematically undervalues the effects of the first and last observations. A modification of the original method, termed the area under the disease progress stairs (AUDPS), has been proposed (Simko and Piepho 2012) that improves the estimation of disease progress by giving a weight closer to optimal to the first and last observations. At approximately evenly spaced evaluations, AUDPS outperforms AUDPC when the average standard deviation of disease scores from the first and last evaluation is smaller than the average standard deviation of scores from all the other evaluations, which is usually the case when scores are recorded from the onset of disease until disease reaches a plateau or is close to it. Since its introduction, the modified method has been used to assess disease progress in many studies, but still less than AUDPC.

Disease-specific R packages ('AUDPC.cal' at https://rdrr.io//forge/plantbreeding/man/ AUDPC.cal.html, Schandry 2017), a stand-alone R function (https://www.apsnet.org/edcenter/ disimpactmngmnt/topc/EcologyAndEpidemiologylnR/DiseaseProgress/Pages/audpc.aspx), and a spreadsheet example (Forbes et al. 2014) are available for calculating AUDPC only. Both the AUDPC and AUDPS methods can be calculated with commonly used statistical software such as SAS (Simko and Piepho 2012) or R ('Agricolae' at https://rdrr.io/cran/agricolae/man/ audps.html and 'Epifitter' at https://cran.r-project.org/web/packages/epifitter/index.html) (Table

${ }^{\dagger}$ Corresponding author: I. Simko; ivan.simko@usda.gov

$\boldsymbol{e}$-Xtra: Supplementary materials are available online.

The author(s) declare no conflict of interest.

Accepted for publication 5 January 2021.

Keywords

data science, AUDPC, AUDPS, area under the curve, calculator 
Table 1. Comparison of the platforms and algorithms for calculating both area under the disease progress curve (AUDPC) and area under the disease progress stairs (AUDPS) values

Features, required skills, and accessibility

AUDPC

AUDPC derivatives ${ }^{a}$

AUDPS

AUDPS derivatives ${ }^{a}$

Descriptive statistics

Graphical output

Correlations with other traits

Epidemic models

Software required

Computer skills

Software accessibility

a Relative and standardized values.

b SAS Institute, Cary, NC.

c R Core Team (2013).

${ }^{d}$ Microsoft Excel is a commercial software, but free alternatives are available (online version of Excel and Google Sheets).

\section{Simko and Piepho (2012)}

$\checkmark$
$\checkmark$
$\checkmark$
$\checkmark$
-
-
-
-
SAS $^{b}$

Intermediate

Commercial

\section{Epifitter}

$\checkmark$

$\checkmark$

$\checkmark$

$\bar{s}$

$\checkmark$

$\checkmark$

$\mathrm{R}^{\mathrm{C}}$

Advanced

Open source

\section{Agricolae}

4
$\checkmark$
$\checkmark$
$\checkmark$
$\checkmark$
$\checkmark$
$\checkmark$
-2
-2

Advanced

Open source
IdeTo

2
-
2
2
2
2
2
2

Excel or Google Sheets Fundamental Commercial or free

Table 2. Area under the disease progress metrics calculated by IdeTo

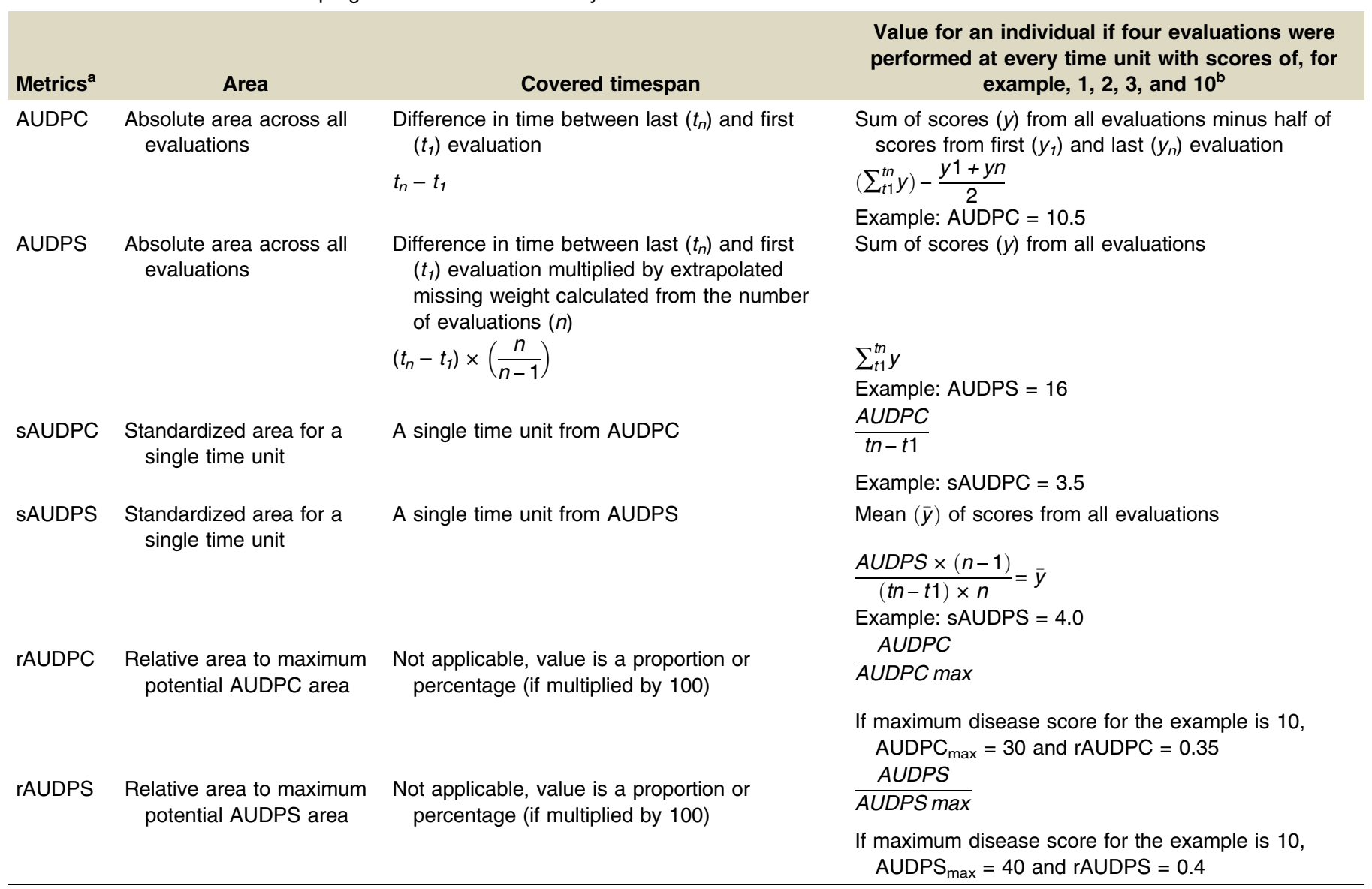

a $A$ UDPC $=$ area under the disease progress curve and AUDPS = area under the disease progress stairs. Though absolute, standardized (s), and relative ( $r$ ) values calculated by a method differ, the coefficient of determination between values obtained by the same method (e.g., AUDPS, sAUDPS, and rAUDPS) from the same dataset always equals $1\left(R^{2}=1\right)$.

${ }^{b}$ An example with four evaluations performed at every time unit is provided to demonstrate the difference in results calculated by the two methods (AUDPC versus AUDPS). In this simple example, the scores recorded for four successive evaluations were 1, 2, 3, and 10, with the maximum potential score of 10. Note that formulas (and related explanations) for calculating AUDPC and AUDPS apply only for evaluations performed at every time unit. If evaluations were not performed at every time unit, formulas for AUDPC and AUDPS are more complex; for detailed explanation, see Simko and Piepho (2012). 


\section{Data input}

1 Entering disease scores for up to 200 individuals evaluated at up to 200 time points.

\section{Results}

Display calculated values of AUDPC, AUDPS, and their derivatives for each individual. Basic statistical description of the data set is provided, including mean, standard deviation, standard error, median, range,

2 minimum and maximum. The progression of disease scores over time and the distributions of AUDPC and AUDPS values in the dataset are presented graphically.

Calculates Spearman and Pearson correlation coefficients between AUDPC and AUDPS values.

\section{Trait correlations}

Entering values for up to five additional traits for the same individuals that were evaluated for disease progress.

Calculates Spearman and Pearson correlation coefficients between AUDPC/AUDPS and the five traits. Shows graphical interpretation of the relationship between the traits and AUDPC and AUDPS values.

Figure 1. Infographic illustrating the content of spreadsheets and the analyses performed by IdeTo calculator. Spreadsheets named (1) Data input, (2) Results, and (3) Trait correlations are used to enter data, calculate area under the disease progress curve (AUDPC) and area under the disease progress stairs (AUDPS) values and their derivatives, perform correlation analyses, and graphically present results. Instructions for use and recommended citation of the IdeTo calculator are provided in spreadsheets (4) Information and (5) Citation. 
1). In addition to calculating AUDPC and AUDPS and their derivatives, these algorithms provide descriptive statistics for the set of values (Agricolae), graphical output (Epifitter and Agricolae), and correlations with other traits (Agricolae), and allow fitting of epidemic models (Epifitter). However, all of the currently available software for calculating AUDPS may be costly (SAS), require knowledge of running command-line scripts, or involve a relative long learning curve. The present article describes IdeTo (meaning "it works" in Slovak), a simple spreadsheet calculator that can be a useful alternative for researchers not using SAS or R.

IdeTo (Supplementary Material S1) is an Excel-based calculator that calculates AUDPC, AUDPS, and their standardized (sAUDPC and SAUDPS) and relative (rAUDPC and rAUDPS) forms (Table 2) according to formulas published by Simko and Piepho (2012). The calculator allows entering data for up to 200 individuals evaluated at 200 timepoints (an individual is a single "item" for which the calculation needs to be performed; it could be a single sample, an average of several samples, a replicate, or a treatment, depending on the experiment). IdeTo is organized into five spreadsheets named "Data input', "Results", "Trait correlations", "Information", and "Citation" (Fig. 1). Data for calculating AUDPC and AUDPS are entered into the Data input spreadsheet with timepoints (e.g., hours, days, weeks, and so on) entered in the first column and disease scores for each individual in the subsequent columns. All values for time series and disease scores must be numerical and missing values are not allowed. Timepoints in time series data have to be entered in ascending order. The calculator performs only a simple check for missing and nonnumerical values and looks for ascending order of timepoints.

Calculated values of AUDPC, AUDPS, SAUDPC, SAUDPS, rAUDPS, and rAUDPS for each individual are shown in the Results spreadsheet. This spreadsheet also provides some basic statistical description of AUDPC and AUDPS values for the set of analyzed individuals, including mean, standard deviation, coefficient of variation, standard error, median, minimum, maximum, and range. The progression of disease scores over time and the distributions of AUDPC and AUDPS values in the dataset are presented graphically. Moreover, results are provided for the Pearson and Spearman correlation coefficients between AUDPC and AUDPS.

The Trait correlations spreadsheet is intended to calculate correlations between the AUDPC or AUDPS values and other traits (up to five traits). Data for all individuals have to be numerical and entered in the same order as in the Data input spreadsheet. Pearson and Spearman correlation coefficients and their $P$ values are shown for the traits and the respective AUDPC and AUDPS values. The graphical representations of the Pearson linear correlations between the traits and the AUDPC and AUDPS values are also displayed in the spreadsheet. The Information spreadsheet provides description of the calculator, while the proposed citation of IdeTo calculator is shown in the Citation spreadsheet.

The spreadsheet calculator works with Microsoft Excel (Microsoft, Redmond, WA) and has been tested on the most recent (as of December 2020) versions of Excel for Mac OS, Windows OS, and Excel Online. All IdeTo calculations can also be performed with Google Sheets (Google, Mountain View, CA); however, the graphical presentation of results is not compatible with this spreadsheet program. The latest version of IdeTo can be downloaded from the figshare repository at https://doi.org/10.6084/m9.figshare.13452107.

\section{Acknowledgments}

IdeTo comes with absolutely no warranty. The mention of trade names or commercial products in this publication is solely for the purpose of providing specific information and does not imply recommendation or endorsement by the U.S. Department of Agriculture (USDA).

\section{Literature Cited}

Forbes, G. A., Perez, W., and Andrade-Piedra, J. 2014. Field Assessment of Resistance in Potato to Phytophthora infestans. International Cooperators Guide. International Potato Center, Lima, Peru.

Jeger, M. J. 2004. Analysis of disease progress as a basis for evaluating disease management practices. Annu. Rev. Phytopathol. 42:61-82.

Jeger, M. J., and Viljanen-Rollinson, S. L. H. 2001. The use of the area under the disease-progress curve (AUDPC) to assess quantitative disease resistance in crop cultivars. Theor. Appl. Genet. 102:32-40.

Lebeda, A., and Jendrulek, T. 1988. Application of method of multivariate analysis in comparative epidemiology and research into field resistance. J. Plant Dis. Prot. 95: 495-505.
Madden, L. V., Hughes, G., and van den Bosch, F. 2007. The Study of Plant Disease Epidemics. American Phytopathological Society, St. Paul, MN.

R Core Team. 2013. R: A Language and Environment for Statistical Computing. R Foundation for Statistical Computing, Vienna, Austria. http://www.R-project.org/

Schandry, N. 2017. A practical guide to visualization and statistical analysis of R. solanacearum infection data using R. Front. Plant Sci. 8:623.

Simko, I., and Piepho, H.-P. 2012. The area under the disease progress stairs: Calculation, advantage, and application. Phytopathology 102:381-389.

Van der Plank, J. E. 1963. Plant Diseases: Epidemics and Control. Academic Press, New York, NY.

Xu, X. 2006. Modelling and Interpreting Disease Progress in Time. Pages 215-238 in: The Epidemiology of Plant Diseases, 2nd Ed. B. M. Cooke, D. G. Jones, and B. Kaye, eds. Springer, Dordrecht, The Netherlands. 